\title{
Measurement and Spatial Difference Analysis on the Accessibility of Road Networks in Major Cities of China
}

\author{
Xiaorui Zhang ${ }^{1,2, *}$, Andong Ren ${ }^{1}$, Lihua Chen ${ }^{3}$ and Xianyou Zheng ${ }^{3}$ \\ 1 Department of Urban Planning, Hefei University of Technology, Hefei 230009, China \\ 2 Laboratory of Digital Human Habitat Studies, Hefei University of Technology, Hefei 230009, China \\ 3 Department of Architecture, Hefei University of Technology, Hefei 230009, China \\ * Correspondence: rgdhf@hfut.edu.cn
}

Received: 10 May 2019; Accepted: 1 August 2019; Published: 4 August 2019

\begin{abstract}
Given the current lack of accessibility research on road networks in 36 major cities in China, the accessibility and its spatial difference were measured by using space syntax and Moran index. The purpose is to provide an important decision-making basis for the Chinese government to grasp the accessibility level of China's urban roads in general and formulate urban traffic development policies. The results show that the mean value of the global integration average is only 1.0009, indicating that the accessibility level is not optimistic in general. The accessibility of 36 major cities was divided into four levels: very low, low, high, and very high. Only four cities, namely Beijing, Shijiazhuang, Xi'an, and Zhengzhou, were at a very high level. The spatial differences of accessibility presented a spatial pattern of "middle-high, east-middle, and west-low". The global integration average, intelligibility, and synergy had significant global spatial autocorrelation, while the local spatial agglomeration distributions of these three indexes were dominated by high-high types. The five cities of Beijing, Shijiazhuang, Hohhot, Taiyuan, and Zhengzhou constituted the core area of high-value clustering of local spatial autocorrelation and presented a spatial form of inverted " $\mathrm{T}$ " shape. The research still has some limitations. The reasons for the low accessibility of developed cities, such as Guangzhou and Nanjing, are worth further analysis. Besides, the main possible influencing factors affecting accessibility, such as urban road density and spatial form, are also worthy of further analysis.
\end{abstract}

Keywords: major cities in China; road network; accessibility; measurement; spatial difference

\section{Introduction}

Road network is the backbone of urban space development and construction by supporting the flow of people and vehicles as the basic channel; it plays a critical and core role in urban economic and social development. Since Hansen first proposed the concept of traffic "accessibility" in 1959, that is, the size of interaction chance between nodes in road network and travel convenience from departure to destination [1], traffic accessibility research has rapidly become an important part of urban and regional planning, traffic planning, urban geography, traffic geography, and other related disciplines [2]; it is also a core index to evaluate the degree of traffic network accessibility, and the higher the value, the better the connectivity of the road network will be [3]. If the accessibility of urban road network is worse, it can lead to urban congestion and large emissions of automobile exhaust, and even increase the risk of air pollution. This poses a serious challenge to the sustainable development of urban ecological environment. On the other hand, accessibility reflects the relative location value of a certain location within a city and the convenience of integration into social and economic activities from the perspective of economic and social development [4]. The development of the urban economy and society is 
inseparable from the flow of people, vehicles, and various material resources in space. Without the support of the urban road network, the process of production, consumption, and transportation in the urban economy and society cannot be successful. If the accessibility of urban road network is worse, it will increase the time cost, transaction cost, and production cost of travel and transportation, which will reduce the opportunity of economic and social exchanges among different people and regions, and ultimately reduce the operational efficiency of the urban economic and social system and the ability of the city to create various kinds of wealth. This also poses a great challenge to the sustainable development of the urban economy and society. Therefore, the accessibility level of a road network is an important factor affecting the sustainable development of cities.

To this day, rich achievements have been made in traffic accessibility studies, including basic theory research $[5,6]$, spatial patterns and evolution analysis of accessibility $[7,8]$, relevant influencing mechanisms of accessibility [9], and technical method of accessibility measurement [10-12]. Accessibility measurement is the core issue, and other related research should be based on measurement. Although accessibility has been thoroughly studied in the past 60 years, it has not yet been unified in the accessibility measurement method. The current methods mainly represent accessibility by calculating the travel time, such as the shortest travel time and the weighted average travel time [13]. Since the 1980s, Bill Hiller has put forward and developed the theory of Spatial Syntax [14,15]. After entering the 21st century, especially since 2010, the theory of Spatial Syntax has been introduced into the study of accessibility measurement of the urban road network and has achieved rich results [16-21]. As a new method of accessibility measurement, Spatial Syntax theory provides a new measurement model. It should be pointed out that the applications of the Space Syntax theory in the accessibility study mainly focuses on the accessibility of a specific city [22,23], region, and other single space [24,25], but there is still a lack of comparative study on traffic accessibility of multiple cities, such as 36 major cities in China in this paper, by using the Space Syntax techniques. Therefore, as a useful exploration and attempt of accessibility measurement research, this paper applies the Spatial Syntax theory to measure the accessibility of road networks of 36 major cities in China, which will expand the application scope of Spatial Syntax theory in accessibility measurement by extending it from a traditional single city to multiple cities. It is hoped that this will provide a methodological reference for the study of accessibility measurement based on Spatial Syntax.

For Chinese cities, with the rapid increase of urban area and population, the accessibility level of China's urban road network is not optimistic. Urban congestion caused by poor accessibility has become one of the important problems that restrict the sustainable development of Chinese cities. Automobile exhaust has become one of the main sources of urban air pollution in China, which has brought serious harm to human health and urban ecological environment [26]. When traffic congestion occurs, the time of vehicle exhaust emission is greatly prolonged, and air pollution is more intense. Urban traffic congestion and air pollution show a high degree of correlation [27]. The Chinese government has attached great importance to the accessibility of urban road networks in recent years. In February 2016, the Central Committee of the Communist Party of China and the State Council issued Some Opinions on Further Strengthening the Management of Urban Planning and Construction, which clearly put forward the need to "form a complete road network and improve road accessibility"; it can be seen that the accessibility of the urban road network has been highly valued at the national level.

Based on this understanding, this paper presents a study on the accessibility of 36 major cities in China, using the Space Syntax technology to quantitatively measure the accessibility of each urban road network, analyze the characteristics of spatial differences of accessibility, and then put forward strategies and suggestions to improve accessibility. The research can not only enrich the content system of traffic accessibility research but also provide decision-making information regarding the sustainable development of urban traffic planning and construction in China. The methods part of the paper gives the principle and calculation formula of Space Syntax techniques. The case study part analyzes the road network accessibility level and spatial difference of 36 major cities in China. The conclusion part gives the research conclusion and points out the future research direction. 


\section{Methodology}

Providing a physical carrier for human spatial movement is the fundamental purpose of the urban road network. The relationship between road, space, and people is the focus of accessibility study; it also constitutes an integrated unity of subjectivity and objectivity. The Space Syntax theory is a classical theory to explore and characterize this relationship [28-30]. The spatial syntactic model subtly represents the relationship between roads, space, and people through a series of measurable quantitative indicators. Commonly used Space Syntax indicators include $C_{i}$ (Connection), $I_{i}$ (Integration), Intelligibility, and Synergy. Where $C_{i}$ is the connection of the $i$ th cell space, which is equal to the number of other cell spaces connected with $i$. The larger the $C_{i}$, the closer the relationship between $i$ th space and other spaces, and the better is the accessibility.

To calculate the Integration degree, the Depth value must be calculated first. The Depth $D$ is an important intermediate variable for calculating the integration index; it refers to the minimum number of connection steps (the shortest distance) of a certain unit space to all other unit spaces. The Mean Depth value $M D_{i}$ is usually used to represent the average minimum number of connecting steps from unit space $i$ to all other unit spaces, the calculation formula is:

$$
M D_{i}=\frac{\sum_{j=1, j \neq i}^{n} d_{i j}}{n-1}
$$

where the numerator represents the total Depth value of unit space $i, d_{i j}$ represents the Depth of $i$ to $j$ $(j \neq i)$, namely the minimum number of connection steps from $i$ to $j$. The sum of the minimum number of connection steps from $i$ to all other nodes is the total Depth of $i$. $n$ is the total number of nodes in the urban space, and $(n-1)$ indicates that at most $(n-1)$ nodes are connected with $i$. Moreover, Integration degree $I_{i}$ is the most commonly used and most effective syntax indicator, and it represents the degree of aggregation or dispersion between a unit space and all other spaces or parts of space within a few steps from it. The Integration degree and Mean Depth are greatly affected by the number of nodes in space. To eliminate the interference of redundant nodes, Space Syntax standardizes Mean Depth with relative asymmetric value $\left(R A_{i}\right)$ and real relative asymmetric value $\left(R R A_{i}\right)$.

$$
\begin{aligned}
R A_{i} & =\frac{2\left(M D_{i}-1\right)}{n-2} \quad R R A_{i}=\frac{R A_{i}}{D_{n}} \\
D_{n} & =\frac{2\left\{n\left[\log _{2}\left(\frac{(n+2)}{3}\right)-1\right]+1\right\}}{(n-1)(n-2)}
\end{aligned}
$$

where $M D_{i}$ is the Mean Depth and $n$ is the total number of nodes in the urban spatial structure. The reciprocals of $R A_{i}$ and $R R A_{i}$ are used to represent the Global Integration degree $I_{i}$ and the Local Integration degree $L I_{i}$ of the unit space $I$, to conform to the conventional rule of "the larger the value, the greater the integration degree", namely:

$$
I_{i}=\frac{1}{R A_{i}} \quad L I_{i}=\frac{1}{R R A_{i}}
$$

The degree of Integration reflects the accessibility and convenience of the space unit and the ability to gather attractive flow and logistics, which in turn reflects the centrality of a unit space relative to the rest of the unit space. When the degree of Integration is greater than 1, and the value is larger, the accessibility and agglomeration of the space are stronger, and vice versa. Besides, the Integration core is also an important concept, which is defined as adding the Integration $I_{i}$ of all unit spaces to obtain a total value $I$, and sorting $I_{i}$ in descending order, from the maximum value begins to accumulate downwards, when the accumulated value reaches at least $5 \%$ of the total value $I$, the unit space set participating in the accumulation at this time can constitute an integrated core. In theory, the integrated 
core has the strongest attraction in the urban space and will be the most accessible area in the urban transportation network.

There is a correlation between local variables $\left(C_{i}, L I\right)$ and the overall variable (I), that is, space with good local accessibility has higher overall accessibility. To quantitatively describe the relationship between part and the whole, Space Syntax provides two evaluation indicators of Intelligibility and Synergy. Intelligibility is the discriminant coefficient $\left(R^{2}\right)$ of the linear fitting equation of Global Integration $(y)$ and Connection $(x)$. The higher the value, the easier the space is understood by the person walking through it, and the more it is possible to infer the overall structural features of the space from the locally acquired spatial connection information (the number of other spaces seen in the local space that intersect with the space), and the easier it is to form a humanized, recognizable spatial structure. Synergy is the discriminant coefficient $\left(R^{2}\right)$ of the linear fitting equation of Global Integration degree $(y)$ and Local Integration degree $(x)$. The higher the value, the overall space is perceived by local space accessibility. The greater the ability to communicate, the more the local spatial structure helps to establish a global picture of the entire space system. In general, the degree of Intelligibility and Synergy is greater than 0.5, the Intelligibility and Synergy are better, and vice versa.

The above-mentioned spatial syntactic indicators represent the functional relationship between the urban road networks and their ability to bring together people flow and vehicles flow, which further forms the level of urban traffic accessibility. The spatial syntactic indicator is an inherent attribute of the urban road network. Through revealing this inherent attribute, we can analyze the social attributes, such as accessibility, convenience, and agglomeration, of urban traffic. Among the spatial syntactic indicators, connection value is the most critical one. It is the basis of calculating other syntactic indicators, and its value affects all other syntactic indicators. If the connection value of a road is greater than 1 , it indicates that it is connected to at least one other road. Generally, road with high connection value has high integration, which is a road network system with good intelligibility, and vice versa. At the same time, as an objective quantitative value, the Integration degree is the core index. Like the traditional travel time index, the Integration degree provides a new way to represent the accessibility level of the urban road network. Generally, if the Integration degree of a road is greater than 1, it indicates that the level of accessibility is good, and the larger the value, the better the accessibility. When the Integration degree is less than 1, the accessibility is poor, and the smaller the value, the worse the accessibility.

In summary, the key to accessibility measurement by using Spatial Syntax lays in calculating the Integration degree index of each road. The general calculation method and process can be summarized as follows: Firstly, the Mean Depth value is calculated by formula (1); secondly, the standardized Mean Depth values are obtained by formula (2) and formula (3); Finally, the formula (4) is applied to calculate the Global Integration degree $I_{i}$ and the Local Integration degree $L I_{i}$, thus realizing the quantitative measurement on the accessibility of the urban road networks.

\section{Study Area and Data}

The study presented in this paper took the road networks of the built-up area in the central districts of 36 major cities in China as the research object, including four municipalities, five municipalities with independent planning status, and 27 provincial capital cities (See Table 1 for details). These 36 cities have accumulated $23 \%$ of the national population with a land area of $5.5 \%$ of the national land size, creating $40 \%$ of the national GDP. As a whole, 36 cities are the concentrated representative of 656 cities in China [31]. Each of them is the central city in the region, even at the national level, and plays a central leading role in national and regional economic and social development. At the same time, the road network planning and construction of these 36 cities are also the targets of the Chinese government's key monitoring [32], but there is still a lack of quantitative measurement and comparative analysis of the accessibility of the road networks in these 36 cities. 
Table 1. List of 36 major cities in China.

\begin{tabular}{cc}
\hline Types & City \\
\hline Municipalities & Beijing, Tianjin, Shanghai, Chongqing \\
Municipalities with Independent planning status & Dalian, Qingdao, Ningbo, Shenzhen, Xiamen \\
& Shijiazhuang, Taiyuan, Hohhot, Shenyang, \\
& Changchun, Harbin, Nanjing, Hangzhou, Hefei, \\
Provincial capital & Fuzhou, Nanchang, Jinan, Zhengzhou, Wuhan, \\
& Changsha, Guangzhou, Nanning, Haikou, Chengdu, \\
& Guiyang, Kunming, Lhasa, Xi'an, Lanzhou, Yinchuan, \\
& Xining, Urumqi \\
\hline
\end{tabular}

From the perspective of urban sustainable development, 36 major cities in China, which have been taken as the research object, are typical and representative. As mentioned above, the accessibility level of a road network is an important factor affecting the sustainable development of cities. This is particularly remarkable in 36 cities. Compared with other cities in China, these 36 cities are subject to different degrees of traffic congestion and air pollution caused by poor accessibility. More importantly, behind the poor accessibility and traffic congestion is the huge waste of energy and the aggravation of pollution. "Poor accessibility-Congestion-Waste-Pollution" has formed a vicious circle in these 36 cities. This not only causes the continuous deterioration of the urban ecological environment but also leads to the decline of various functions of urban economy and society, which has become one of the main obstacles to the sustainable development of 36 cities. When accessibility is poor, traffic congestion is more likely to occur and more exhaust will be generated, which can further aggravate urban air pollution and affect the sustainable development of urban ecological environment. Taking $\mathrm{PM}_{2.5}$, the most common urban air pollutant in China, as an example, automobile exhaust emissions have become an important source of $\mathrm{PM}_{2.5}$ in 36 cities. The automobile exhaust emissions in Beijing, Shanghai, Hangzhou, Jinan, Guangzhou, and Shenzhen are the primary sources of $\mathrm{PM}_{2.5}$, accounting for $45.0 \%, 29.2 \%, 28.0 \%, 32.6 \%, 21.7 \%$, and $52.1 \%$, respectively. The automobile exhaust emissions in Nanjing, Wuhan, Changsha, Ningbo, and Shijiazhuang are the second-largest sources of $\mathrm{PM}_{2.5}$, accounting for $24.6 \%, 27.0 \%, 24.8 \%, 22.0 \%$, and $15.0 \%$ respectively. In the other 25 cities, the proportion of automobile exhaust emissions to the sources of $\mathrm{PM}_{2.5}$ is all $10 \%$ or more [33]. Besides, the poor accessibility can lead to the decline of the average speed of urban automobiles, thus affecting the operational efficiency of urban economy and society and bringing about certain economic losses, which can restrict the sustainable development of urban economy and society. In the central areas of 36 cities, the average speed of automobiles is less than $25 \mathrm{~km}$ per hour due to poor accessibility and congestion, which is much lower than the design speed of 40-60 km per hour of urban roads in China. Meanwhile, the annual economic loss per capita in 36 cities caused by poor accessibility and congestion is about 4000-12,000 Yuan, of which Beijing has the largest value of 11,747 Yuan, and Urumqi has the smallest value of 4273 Yuan [34]. Therefore, using the Space Syntax technology to quantitatively measure the accessibility of road networks in the 36 major cities and analyze their spatial pattern characteristics have an important guiding value for the planning and construction of China's urban road transportation system and sustainable development. It can provide an important decision-making basis for the Chinese government to grasp the accessibility level of China's urban roads in general and formulate urban traffic development policies. It also provides a reference for other cities' traffic accessibility research.

The study adopted the axis map model in Space Syntax analysis. First, the 2018 China Major Urban Road Network Density Monitoring Report released by the Ministry of Housing and Urban-Rural Development in April was taken as the basic data source; the road network status map of each city was extracted; the spatial projection transformation in ArcGIS, spatial correction, and other processing was performed; then the raster maps of the 36 urban road networks were obtained. Secondly, the axial map vector data according to the grid map were digitized, and the topology check to ensure that there was 
no independent axis was performed. Finally, after the inspection, according to the spatial syntactic calculation principle of the above formulas (1) (4), the spatial syntactic index measurements and their visual expressions of the 36 urban road networks were obtained; thus the accessibility level of major urban road networks in China was quantitatively measured.

\section{Analysis of Results}

\subsection{Overall Analysis}

The results of the main syntactic indicators of the road networks of the 36 major cities in China are shown in Table 2. Given a large amount of axis data of the 36 cities, the average of the syntactic indicators was used in the study to represent the level of accessibility in each city. Further, the descriptive statistical values of the 36 city syntax indicators calculated according to data in Table 2 are shown in Table 3. At the same time, according to the numerical value of the Global Integration degree of each road axis, the study used different colors to spatially express it, as shown in Figure 1. The warmer the color was, the greater the Integration value of the road axis was, and the better was the accessibility. Whereas the cooler the color was, the smaller the Integration value of the road axis was, and the worse was the accessibility.

Table 2. The calculation results of the syntactic index of road networks in 36 major cities of China.

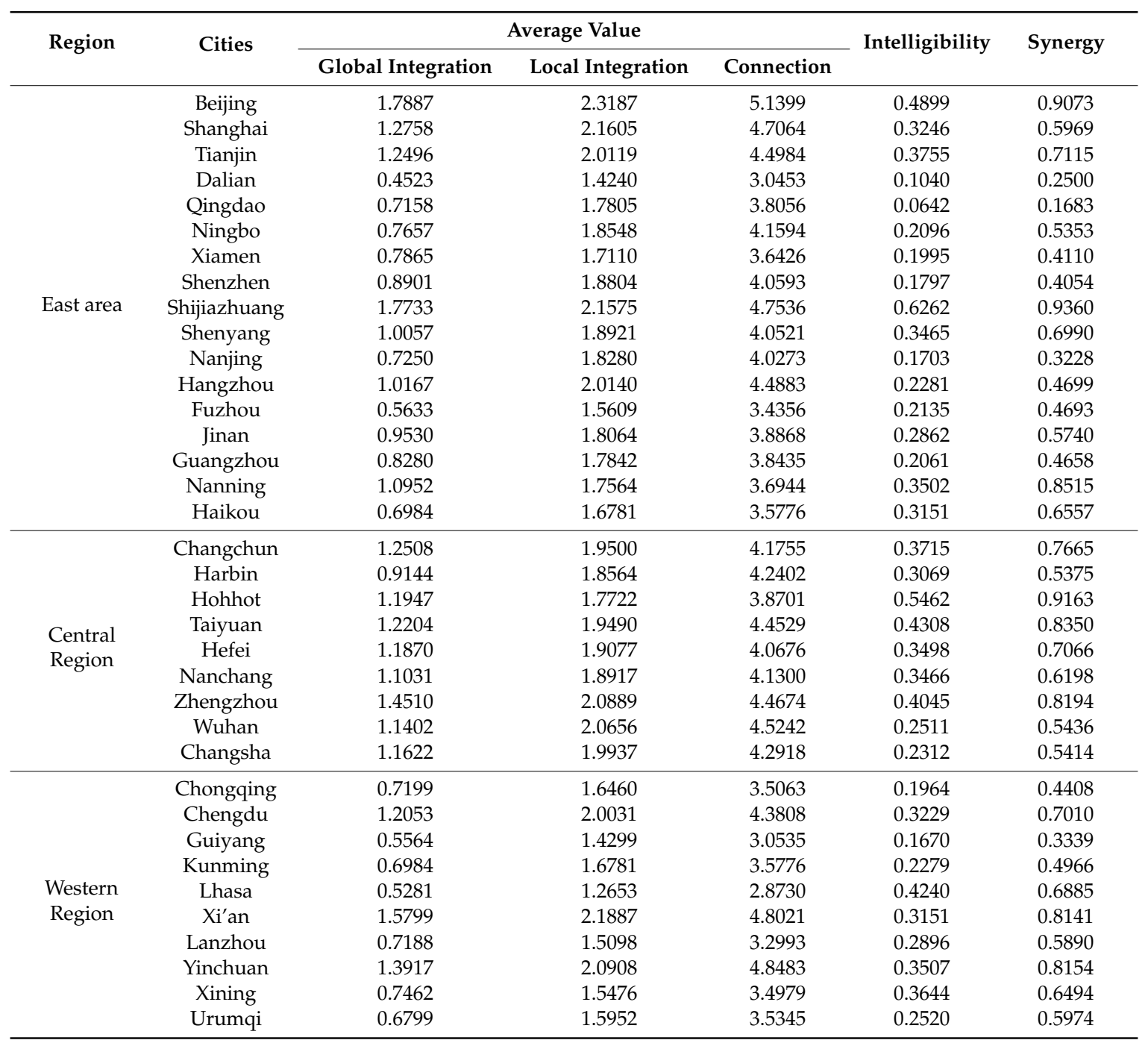




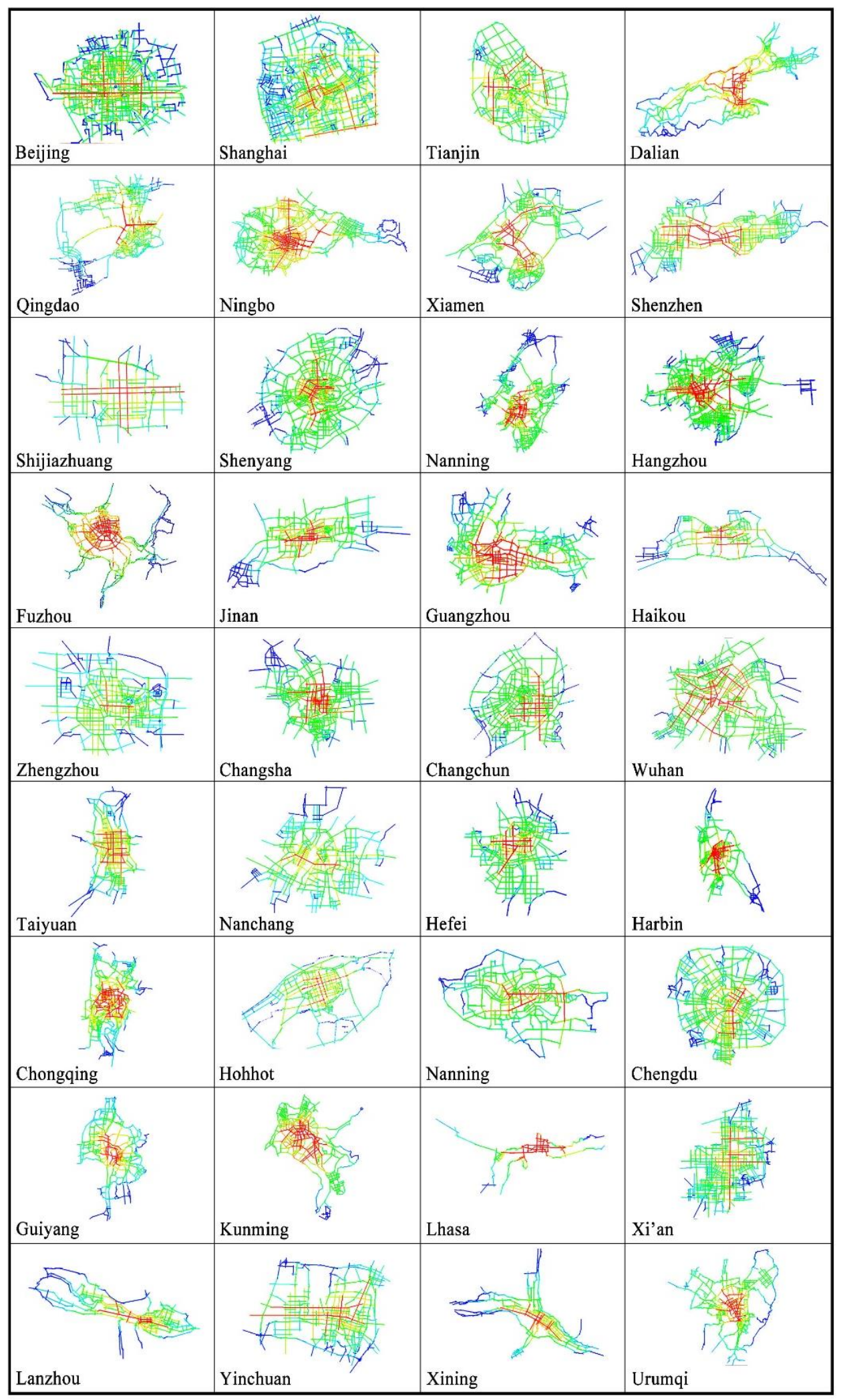

Figure 1. The Global Integration of road networks in 36 major cities of China. 
Table 3. Descriptive statistics of syntactic index of road networks in 36 major cities of China.

\begin{tabular}{ccccc}
\hline Syntactic Index & Average & Minimum & Maximum & Standard Deviation \\
\hline Global Integration & 1.0009 & 0.4523 & 1.7887 & 0.3409 \\
Local Integration & 1.8347 & 1.2653 & 2.3187 & 0.2394 \\
Connection value & 4.0114 & 2.8730 & 5.1399 & 0.5491 \\
Intelligibility & 0.3011 & 0.0642 & 0.6262 & 0.1175 \\
Synergy & 0.6067 & 0.1683 & 0.9360 & 0.1915 \\
\hline
\end{tabular}

As illustrated in Table 2, Table 3, and Figure 1, it is clear that the syntactic indicators of the road networks in the 36 major cities in China varied widely. The maximum values of the syntactic indicators (GI, LI, C, Intelligibility, Synergy) were 3.95 times, 1.83 times, 1.79 times, 9.75 times, and 5.56 times of the minimum values, respectively, and the difference was significant. The average values of the Global and Local Integration values were 1.0009 and 1.8347, both greater than one, indicating a good level of accessibility overall. The average value of the Connection was 4.0114, which reflected the average number of road axes connecting other road axes in China's major cities, which is about 4, with Lhasa having the smallest average Connection value (2.8730), and Beijing having the largest (5.1399). The average of the Intelligibility was 0.3011 , which was significantly less than 0.5 , indicating that the road space of these cities is generally more difficult to recognize. Qingdao had the smallest Intelligibility (0.0642), while Shijiazhuang had the largest (0.6262), and the maximum was 9.75 times the minimum, which was the biggest difference among the five syntactic indicators. At the same time, only two cities (Shijiazhuang, Hohhot) had an Intelligibility of more than 0.5 , which means that as the size of the city expands, the road space becomes more and more complex and more difficult to be perceived by people. The average degree of Synergy was 0.6067 , which reflects a good correlation and integration of urban local space and overall space. Like Intelligibility, Qingdao has the smallest degree of Synergy (0.1683), and Shijiazhuang had the largest (0.9360), while a total of 25 cities had a Synergy greater than 0.5 . The Intelligibility and Synergy index values are worthy of attention in five cities (Shijiazhuang, Hohhot, Beijing, Qingdao, Dalian), which had the same ranking in terms of Intelligibility and Synergy, among them, Shijiazhuang, Hohhot, and Beijing had the highest Intelligibility and Synergy, ranked on the top three in succession; Qingdao had the lowest Intelligibility and Synergy, followed by Dalian.

In the technical method system of Space Syntax, Global Integration is the most commonly used and most effective indicator, which can more accurately and intuitively characterize the overall accessibility level of the urban road networks compared to other indicators [15], so it should be a focus to analyzing the degree of Global Integration. In the Global Integration, although the average value (1.0009) was slightly larger than one, 18 cities were having Global Integration value greater than one and also 18 cities having Global Integration value less than one, which reflects that the accessibility of road networks in major cities in China is not optimistic. In terms of specific values, Dalian had a minimum of 0.4523 , Beijing had a maximum of 1.7887, and Shijiazhuang had a slight gap (1.7733) from the maximum. Further, with "1.0" as the basic demarcation point and according to the principle of equal spacing, the Global Integration of 36 cities was divided into four echelons to characterize different accessibility levels, see Table 4 for details.

According to Table 4, there were 7 and 11 cities with low and lower accessibility levels, respectively, and 14 and 4 cities with higher and high accessibility levels, respectively. On the whole, the total accessibility level of 25 cities was lower and higher, and the specific value was between 0.7026 1.3972. The number of cities in four grades showed a typical normal distribution. Based on the above analysis, five cities, including Shijiazhuang, Hohhot, Beijing, Qingdao, and Dalian, need to pay attention. According to Table 4, Dalian, Shijiazhuang, and Beijing can be further found to be unique, and they are at the lowest and highest level of accessibility. Therefore, these three cities are more typical and representative. 
Table 4. Accessibility classification of road networks in 36 major cities of China.

\begin{tabular}{|c|c|c|}
\hline Global Integration Average Value & Accessibility Level & Cities \\
\hline $0.4523 \sim 0.7026$ & low & $\begin{array}{l}\text { Dalian, Lhasa, Guiyang, Fuzhou, } \\
\text { Urumqi, Kunming, Haikou }\end{array}$ \\
\hline $0.7026 \sim 0.9530$ & Lower & $\begin{array}{l}\text { Qingdao, Lanzhou, Chongqing, } \\
\text { Nanjing, Xining, Ningbo, Xiamen, } \\
\text { Guangzhou, Shenzhen, Harbin, } \\
\text { Jinan }\end{array}$ \\
\hline $1.0057 \sim 1.3972$ & Higher & $\begin{array}{l}\text { Shenyang, Hangzhou, Nanning, } \\
\text { Nanchang, Wuhan, Changsha, } \\
\text { Hefei, Hohhot, Chengdu, Taiyuan, } \\
\text { Tianjin, Changchun, Shanghai, } \\
\text { Yinchuan }\end{array}$ \\
\hline $1.3972 \sim 1.7887$ & high & $\begin{array}{l}\text { Zhengzhou, Xi'an, Shijiazhuang, } \\
\text { Beijing }\end{array}$ \\
\hline
\end{tabular}

\subsection{Spatial Difference Analysis}

According to the traditional spatial division of the eastern, central, and western regions in China, based on Table 2, the syntax measurement results of accessibility of major urban road networks in the eastern, central, and western regions of China were further obtained, as shown in Table 5. Based on the results in Tables 2 and 5, it can be found that considering the most important Global Integration (GI) indicators, among the 17 cities of eastern region, seven cities, such as Beijing, Shanghai, Tianjin, and other four cities, had GI greater than one, and the remaining 10 cities had GI less than one; in the central region's nine cities, only Harbin (0.9144) had GI slightly less than one, and the remaining eight cities had GI greater than one. In the 10 cities in the western region, Chengdu, $\mathrm{Xi}^{\prime}$ an, and Yinchuan had GI larger than one, and the remaining seven cities had GI less than one. From the number of cities with index values greater than one, the central region had contributed the most cities. At the same time, from the perspective of intra-regional differences in global integration, the difference between cities in the central region was the smallest, and the standard deviation was only 0.1408 , which was much smaller than 0.3742 in the eastern region and 0.3695 in the western region. On the whole, the accessibility level of the urban road network in the central region was the highest.

Table 5. The analysis of spatial difference of road networks in 36 major cities of China.

\begin{tabular}{ccccccc}
\hline \multirow{2}{*}{$\begin{array}{c}\text { Regional } \\
\text { Space }\end{array}$} & $\begin{array}{c}\text { Number of } \\
\text { Cities }\end{array}$ & $\begin{array}{c}\text { Global } \\
\text { Integration }\end{array}$ & $\begin{array}{c}\text { Local } \\
\text { Integration }\end{array}$ & Connection & Intelligibility & Synergy \\
\hline East & 17 & 0.9755 & 1.8599 & 4.0480 & 0.2758 & 0.5547 \\
Central & 9 & 1.1804 & 1.9417 & 4.2466 & 0.3598 & 0.6985 \\
Western & 10 & 0.8825 & 1.6955 & 3.7373 & 0.2910 & 0.6126 \\
\hline
\end{tabular}

According to Table 5, the spatial differences in the accessibility of the urban road networks in the three major regions of East, Central, and West were obvious, in terms of Global Integration, Local Integration, and Connection; the western region had the lowest accessibility level, the eastern region was in the middle, and the central region had the highest value of the three indicators, indicating that its accessibility level was also the highest. The spatial distribution demonstrated a pattern of "central-high, east-middle, and west-low". In the indicators of Intelligibility and Synergy reflecting the relationship between local and overall, the central region was still the highest, followed by the western region, while the eastern region was the lowest. The analysis indicated that the spatial distribution pattern of "central-high, east-middle, and west-low" was different from the traditional concept of "developed in the east, second in the middle, and backward in the west". The cities in the eastern 
region are economically and socially better developed; the investment in urban transportation facilities is large; the industrial foundation is good, and infrastructure conditions are excellent, and the level of accessibility is supposed to be the highest. However, the result of Space Syntax measurement denies this traditional understanding. The western region is a region with relatively backward economic and social development in China, the construction of urban transportation facilities is relatively lagging, and the lowest level of accessibility is consistent with this analysis. The central region has developed rapidly in recent years under the guidance of the "Rise of Central" national strategy, and the construction of urban transportation facilities is changing with each passing day, and the Space Syntax measurement results also verify this fact.

To further explore the spatial differences in the accessibility of road networks in major cities in China, the spatial autocorrelation of syntactic indicators was analyzed. Spatial autocorrelation analysis is a spatial data analysis method to study whether the observed value of a certain location in space is correlated with those values of its adjacent locations and the degree of correlation [35], to test whether an attribute of a spatial object is adjacent distribution or non-adjacent distribution [36]. The Moran's I index (including Global Moran's I index and Local Moran's I index) is the most commonly used method for calculating spatial autocorrelation. The Global Moran's I index is calculated as follows:

$$
\begin{gathered}
I=\frac{\sum_{i=1}^{n} \sum_{j=1}^{n} w_{i j}\left(x_{i}-\bar{x}\right)\left(x_{j}-\bar{x}\right)}{S^{2} \sum_{i=1}^{n} \sum_{j=1}^{n} w_{i j}} \\
S^{2}=\frac{1}{n} \sum_{i=1}^{n}\left(x_{i}-\bar{x}\right)^{2} \\
\bar{x}=\frac{1}{n} \sum_{i=1}^{n} x_{i}
\end{gathered}
$$

where $I$ is the Global Moran's I index, $n$ is the number of space units, $x_{i}$ and $x_{j}$ are the observation values of the $i$ th space unit and $j$ th space unit, respectively. The $w_{i j}$ is the spatial weight value, indicating the adjacent relation of the $i$ and $j$. If it is adjacent, $w=1$; otherwise, it is 0 . The Global Moran's I index is generally $(-1,1)$. When $I$ is greater than 0 , it indicates positive spatial correlation, and the distribution is spatially clustered. When $I$ is less than 0 , it indicates negative spatial correlation, and the spatial distribution is scattered. The value of 0 indicates that there is no spatial autocorrelation, meaning the spatial distribution is random. Global Moran's I index is a global assessment of spatial autocorrelation that cannot reflect the degree of correlation between a sub-region and its peripheral sub-regions. Thus, local spatial autocorrelation analysis must be conducted. The local indicator of spatial association (LISA) of each observation value is an index reflecting the spatial aggregation of each value with its spatially adjacent observation value [37]. For the ith space unit, the Local Moran's I index is calculated as follows:

$$
I_{i}=\frac{\left(x_{i}-\bar{x}\right)}{S^{2}} \sum_{i, j=1}^{n} w_{i j}\left(x_{j}-\bar{x}\right)
$$

where $I_{i}$ is the Local Moran's I index. The other variables have the same meaning as in formula (5). Besides, the significance of spatial autocorrelation can be tested according to the calculated test statistics. The general significance level is 0.05 . More detailed principles of Moran's I index can be found in the related literature [38-40] and have not been repeated here. Specifically, the Global Moran's I index and Local Moran's I index of each syntactic index value were calculated by using the point data of 36 major cities and the spatial weight method based on distance. The results are shown in Table 6 and Figure 2. 
Table 6. The analysis of globe spatial autocorrelation of road networks in 36 major cities of China.

\begin{tabular}{cccccc}
\hline Spatial Autocorrelation & $\begin{array}{c}\text { Global } \\
\text { Integration }\end{array}$ & $\begin{array}{c}\text { Local } \\
\text { Integration }\end{array}$ & Connection & Intelligibility & Synergy \\
\hline Global Moran's I index & 0.2451 & 0.1289 & 0.1428 & 0.3654 & 0.2902 \\
$p$-value & 0.009 & 0.071 & 0.062 & 0.002 & 0.005 \\
\hline
\end{tabular}
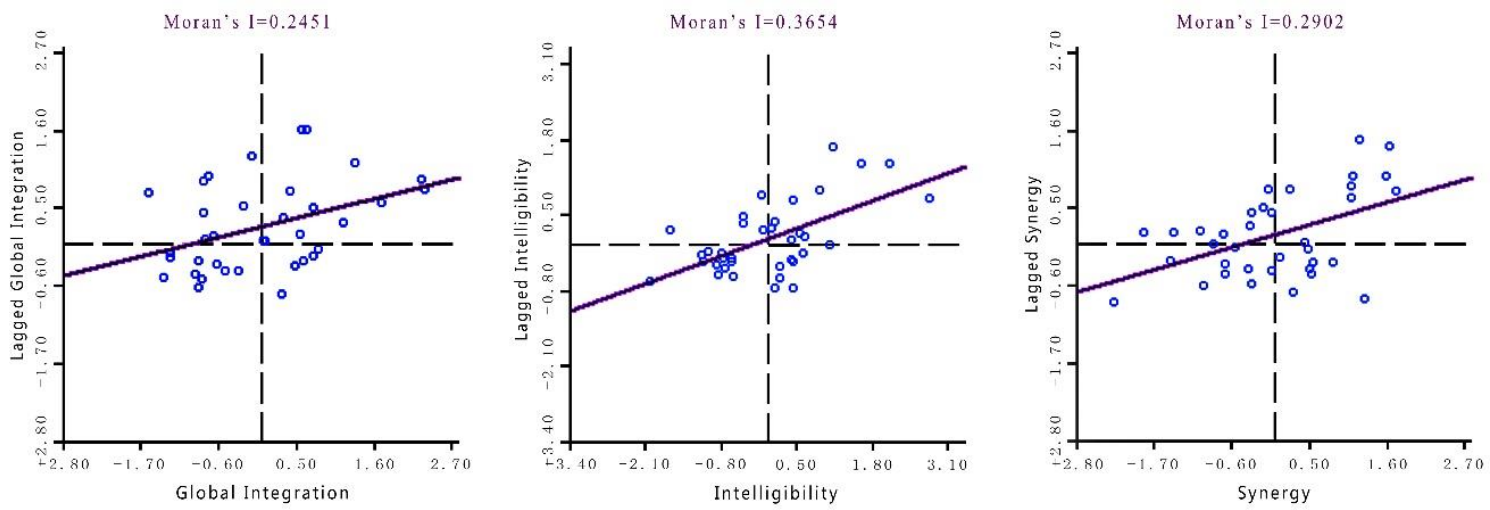

Figure 2. Moran's I scatter graph of the syntactic index of road networks in 36 major cities of China.

Table 6 and Figure 2 show that under the saliency constraint of $p=0.05$, the Global Integration, Intelligibility, and Synergy indicators all pass the test, indicating that there is a significant spatial positive correlation, and the values of Global Moran's I index are sorted into Intelligibility > Synergy > Global Integration. Although the values of Global Moran's I index of Local Integration and Connection were greater than 0 , each $p$-value did not pass the significance test, and the spatial autocorrelation was not significant. Furthermore, the values of Local Moran's I index of Global Integration, Intelligibility, and Synergy were calculated, and the local indications of spatial association (LISA) maps of three indicators were obtained to show the positive spatial correlation with high-high (H-H) and low-low (L-L) or the negative spatial correlation with high-low (H-L) and low-high (L-H) or no significant spatial correlation (spatial randomness) between the research index value and the mean value of its neighborhood index value. The results are shown in Figures 3-5.

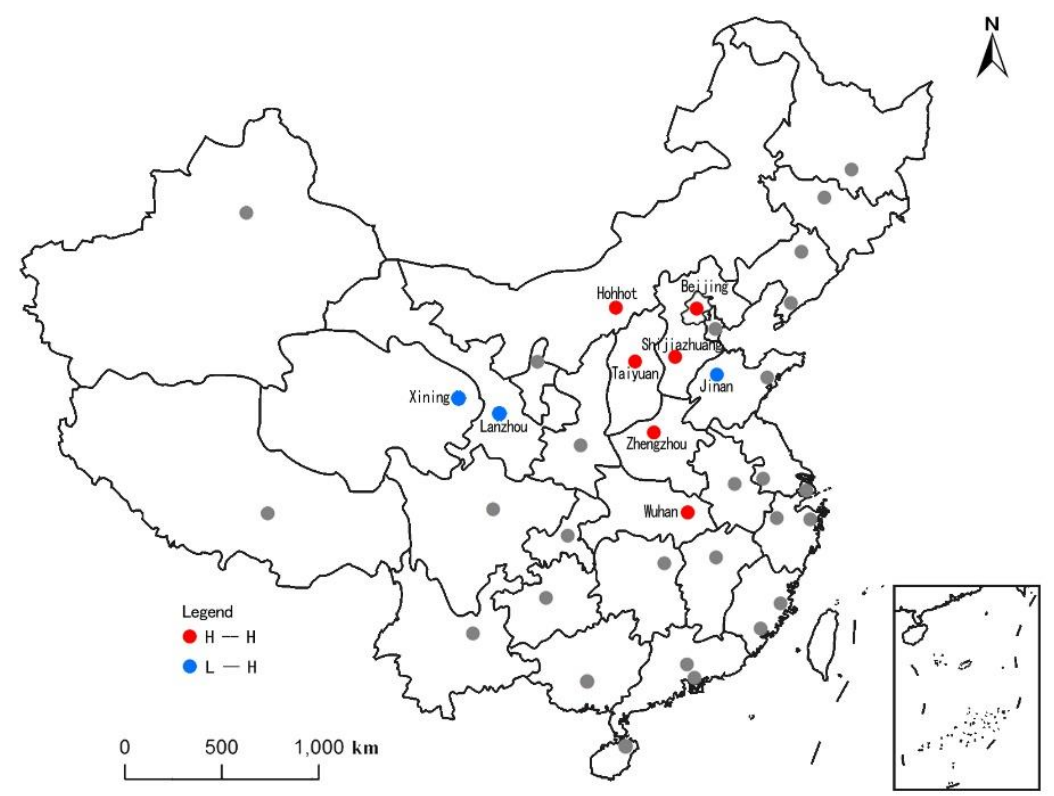

Figure 3. Cluster map of Global Integration with local indications of spatial association. 


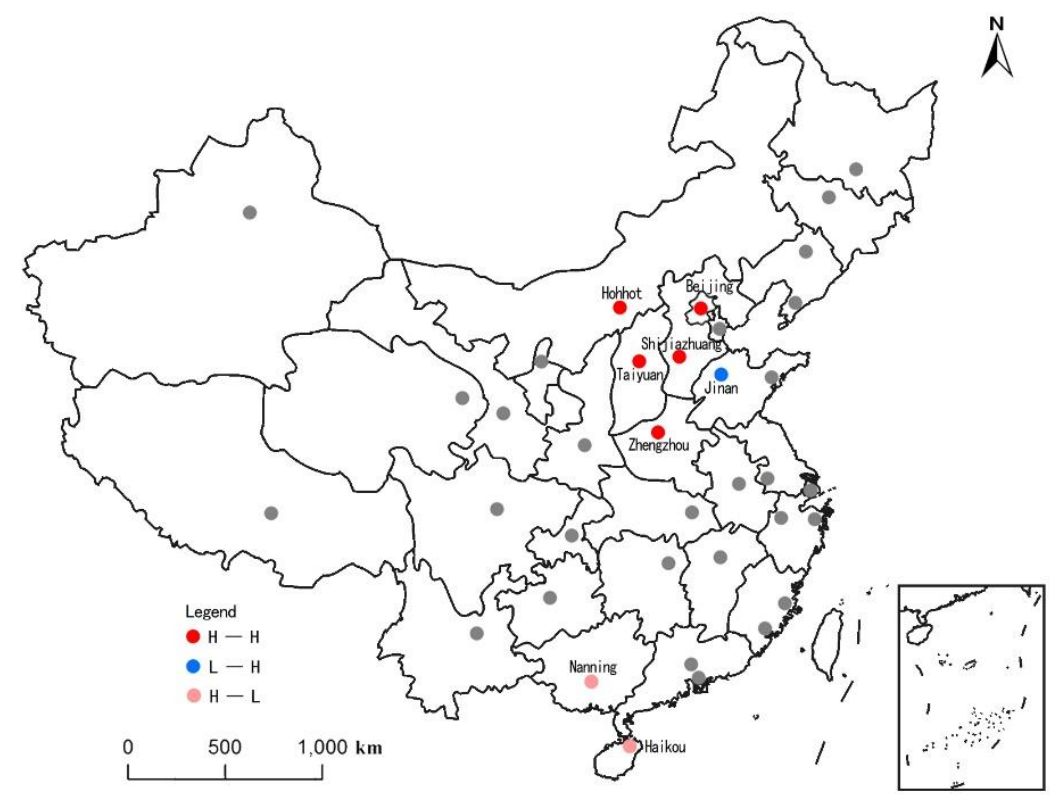

Figure 4. Cluster map of Intelligibility with local indications of spatial association.

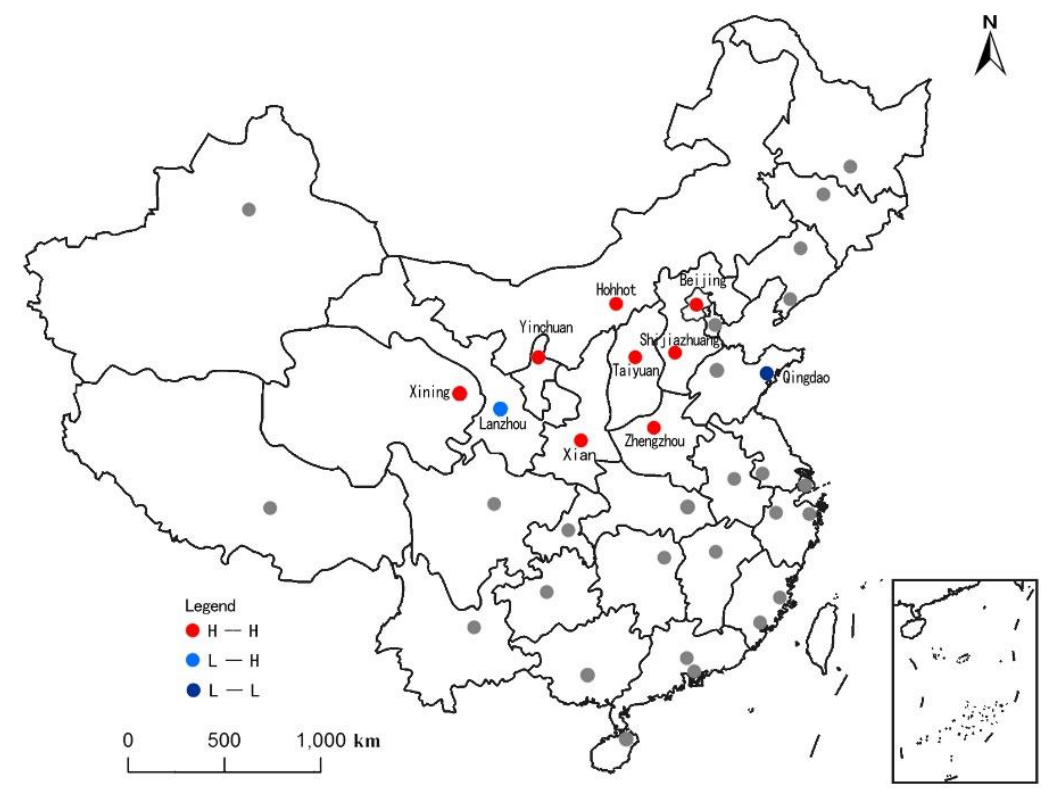

Figure 5. Cluster map of Synergy with local indications of spatial association.

According to Figures 3-5, the three syntactic indicators were mainly high-high type in the local spatial agglomeration distribution, showing a distinct spatial pattern of high-value clustering. In terms of Global Integration, there were six cities, including Beijing, Shijiazhuang, Hohhot, Taiyuan, Zhengzhou, and Wuhan in the high-value areas; in terms of Intelligibility, high-value areas included five cities, namely Beijing, Shijiazhuang, Hohhot, Taiyuan, and Zhengzhou. In terms of Synergy, there were eight cities in the high-value areas, including Beijing, Shijiazhuang, Hohhot, Taiyuan, Zhengzhou, $\mathrm{Xi}^{\prime}$ an, Yinchuan, and Xining. On the whole, Beijing, Shijiazhuang, Hohhot, Taiyuan, and Zhengzhou constituted the core area of high-value agglomeration of local spatial autocorrelation. Hohhot, Taiyuan, and Zhengzhou formed the vertical axis from north to south; Taiyuan, Shijiazhuang, and Beijing formed the horizontal axis from west to east. Together, they formed the spatial pattern of the inverted " $\mathrm{T}$ " shape. 


\section{Discussion}

As the concentrated representative of 656 cities in China, 36 major cities generally reflect the accessibility level of China's urban road network. There is still a lack of quantitative measurement and comparative analysis of the accessibility of the road networks in these 36 cities. Because of this research situation, the accessibility of the 36 major urban road networks in China was measured by using the Space Syntax, and the spatial differences of accessibility were also analyzed by using Moran index in spatial autocorrelation analysis. Therefore, it can provide important decision-making basis for Chinese cities to improve the accessibility level of the road network and realize the sustainable development of urban traffic system. More importantly, this paper explores the application scope of Spatial Syntax theory in accessibility measurement and expands it from the traditional single city accessibility measurement to the accessibility measurement and comparative analysis of multiple cities, which will enrich the theoretical and methodological system of traffic accessibility research.

The research still needs to be further explored in some aspects: the accessibility of some developed eastern cities, such as Guangzhou, Shenzhen, Nanjing, Dalian, and Qingdao, was at a low or lower level, which is deviated from traditional perception, and the reasons behind are worth exploring. The three cities of Dalian, Shijiazhuang, and Beijing were typical and representative in terms of accessibility. The reasons why their accessibility is low and high are still worth further analysis, to provide experience for other cities. This paper only quantitatively measures accessibility, but the possible influencing factors of accessibility, for instance, road density, road length, road area, spatial form, and the relationship between accessibility and these factors, have not been analyzed, which will be another important direction for future research. Despite the limitations, this paper provides a method to measure the accessibility and spatial differences of the 36 major urban road networks in China by the comprehensive application of Space Syntax and spatial autocorrelation analysis, which can be regarded as one useful attempt and exploration in urban traffic accessibility research field. It is believed that with the deepening of the research and the continuous deepening of the accessibility study of major urban road networks in China, some deeper causes and mechanisms will be gradually discovered and analyzed. Accessibility measurement will play a more scientific guiding role in the planning and construction of urban traffic networks.

\section{Conclusions}

The accessibility level of the road network is an important factor affecting the sustainable development of cities. This is particularly remarkable in 36 major cities of China. Research on accessibility measurement and spatial difference analysis of road networks in 36 major cities of China is a critical starting point to improve the accessibility level of the urban road network in China, which has important practical and theoretical significance. The main conclusions are as follows: there are 18 cities whose Global Integration average value is greater than 1 and also 18 cities whose Global Integration average value is less than 1 , which shows that the accessibility level of major cities in China is not optimistic. The road accessibility of 36 major cities is divided into four grades: low, lower, higher, and high, and the number of four grades of cities presents a typical normal distribution. The spatial difference in the accessibility of urban road network in the three regions is obvious, forming a spatial pattern of "central-high, east-middle, and west-low"; the overall accessibility level of the cities in the central region is the highest. There are significant spatial autocorrelations among the Global Integration, Intelligibility, and Synergy index; the local spatial agglomeration distribution is dominated by high-high type. Beijing, Shijiazhuang, Hohhot, Taiyuan, and Zhengzhou, together form the spatial pattern with the inverted " $\mathrm{T}$ " shape of high-value agglomeration.

In the past, Chinese cities paid more attention to enlarging the scale (length and width) of the road network, while the accessibility level of the road network was once neglected. For example, the cities in developed eastern China, such as Nanjing and Guangzhou, already have huge road network scales, but their accessibility levels are not high. At the same time, the congestion and environmental pollution caused by poor accessibility have affected the sustainable development of these cities. Fortunately, the 
Chinese government has realized this problem since 2016. Improving the accessibility of the urban road network has been regarded as an important national policy. Therefore, the study of accessibility measurement and spatial difference analysis of road networks in 36 major cities of China is helpful to provide important decision-making information for the Chinese government to grasp the accessibility level of China's urban road network in general and lay a scientific foundation for the formulation of urban traffic sustainable development policy in the future in China.

Author Contributions: conceptualization, X.Z. (Xiaorui Zhang); methodology, X.Z. (Xiaorui Zhang) and A.R.; software, A.R.; validation, L.C. and X.Z. (Xianyou Zheng); data curation, A.R.; writing-original draft preparation, X.Z. (Xiaorui Zhang); writing-review and editing, L.C.

Funding: This research was funded by the Soft Science Research Project of Hefei City, grant number JS2017HKRK0199; by the Scientific Research Project of the Hefei University of Technology, grant number W2019JSFW0102.

Conflicts of Interest: The authors declare no conflict of interest.

\section{References}

1. Hansen, W.G. How accessibility shapes land-use. J. Am. Inst. Plan. 1959, 25, 73-76. [CrossRef]

2. Wu, W.; Cao, Y.H.; Cao, W.D. The spatial pattern and its evolution of highway network in the Yangtze River Delta. Acta Geogr. Sin. 2006, 61, 1065-1074.

3. Li, T.; Cao, X.S.; Huang, X.Y. Relationship between the spatial pattern of traffic accessibility and population change in the Pearl River Delta. Geogr. Res. 2012, 31, 1661-1672.

4. Liu, H.L.; Bao, A.M.; Chen, X.; Zhang, X.L.; Zhang, J.Y. The effect of transport accessibility on regional economic performance. Acta Geogr. Sin. 2008, 63, 428-436.

5. Kwan, M.P.; Murray, A.T.; O'Kelly, M.E. Recent advances in accessibility research: Representation, methodology and applications. Geogr. Syst. 2003, 5, 129-138. [CrossRef]

6. Chen, J.; Lu, F.; Cheng, C.X. Review on the progress of accessibility measurement methods and applications. Prog. Geogr. 2007, 26, 100-110.

7. Cao, X.S.; Yan, X.P. The impact of the evolution of land network on spatial structure of accessibility in the developed areas: The case of Dongguan city in Guangdong province. Geogr. Res. 2003, 22, 305-312.

8. Zhang, X.; Du, J.; Li, W.Q. Monitoring analysis of traffic network accessibility in Chengdu. Geomat. World 2017, 24, 15-19.

9. Li, Z.; Zhou, S.L.; Wu, S.H. The impact of metro lines on public transit accessibility and land value capture in Nanjing. Acta Geogr. Sin. 2014, 69, 255-267.

10. Ma, X.L.; Ma, Y.J. Analysis of the relationship between urban traffic accessibility and economic development level based on GIS in China. Arid Zone Resour. Environ. 2016, 30, 8-13.

11. Zhang, Z.X.; Li, T.S. Study on the accessibility of county-level traffic based on GIS-Taking Shanxi Province as an example. Hum. Geogr. 2010, 25, 100-104.

12. Huang, J.; Zhong, Y.X.; Li, J.X. Economic accessibility of Chinese capital cities based on high-speed rail network. Geogr. Res. 2016, 35, 757-769.

13. Liu, C.M.; Zhang, Y.G.; Liu, J.; Dong, L.Q. Study on the evolution of the urban comprehensive transportation accessibility and the coordination degree with the economic development. Econ. Geogr. 2011, 31, 2028-2033.

14. Hiller, B.; Hanson, J. The Social Logic of Space; Cambridge University Press: London, UK, 1984.

15. Hiller, B. The hidden geometry of deformed grids: Or why space syntax works, when it looked as though it shouldn't. Environ. Plan. Plan. Des. 1999, 26, 169-191. [CrossRef]

16. Batty, M. Exploring isovist fields: Space and shape in architectural and urban morphology. Environ. Plan. Plan. Des. 2001, 28, 123-150. [CrossRef]

17. Chen, M.X.; Shen, F.; Zha, L.S.; Jin, B.S. A research on urban traffic network based on space syntax: A case study on Wuhu City. Geogr. Geo-Inf. Sci. 2005, 21, 39-42.

18. Chen, Y.S.; Chen, W.C. Study on the road network form of Quanzhou City based on space syntax. Trop. Geogr. 2011, 31, 604-615.

19. Bai, Y.P.; Wu, C.Y.; Chen, B.W. Simulation and analysis of accessibility of complex terrain areas-Taking the economic belt of the northern slope of Tianshan as an example. J. Geogr. Geo-Inf. Sci. 2013, 29, 74-79. 
20. Liu, Y.; Song, R.; Li, Z.J. Evaluating of the accessibility to rail transit based on spatial syntax. Urban Rapid Rail Transit 2014, 27, 70-74.

21. Luo, Z.F.; Xu, J.; Gong, X.; He, X.; Fan, Y.Y. Characteristics analysis of urban road network based on spatial syntax in Lanzhou City. J. Northwest Norm. Univ. (Nat. Sci.) 2018, 54, 105-111.

22. Li, Q.F.; Li, R.J.; Fu, X.Q. Accessibility model and its application for three-dimensional traffic in City based on spatial syntax: A case study of Shijiazhuang. Geogr. Geo-Inf. Sci. 2015, 31, 70-75.

23. Qi, X.Q.; Wu, X.F.; Wang, J.T. A study of three-dimensional traffic city based on spatial syntax-A case study of Harbin. Technol. Econ. Areas Commun. 2016, 18, 16-20.

24. Liang, Y.; Zheng, X.Q.; Song, Q.H. Accessibility evolution of China's transportation network. Geogr. Res. 2017, 36, 2321-2331.

25. Li, C.X.; Gao, X.; He, B.J.; Wu, J.Y.; Wu, K.N. Coupling coordination relationships between urban-industrial land use efficiency and accessibility of highway networks: Evidence from Beijing-Tianjin-Hebei urban agglomeration, China. Sustainability 2019, 11, 1446. [CrossRef]

26. Lin, T.J.; Qiu, D.Q.; Sun, J. A preliminary study on correlation between traffic congestion and air pollution. China Popul. Resour. Environ. 2017, 27, 58-60.

27. Tian, Z.Q. Foreign experts recruit to deal with traffic congestion and environmental pollution. Road Traffic Manag. 2012, 4, 56-57.

28. Zhang, X.R.; Cheng, Z.G.; Bai, Y. Progress and Prospects of Space Syntax Research. Geogr. Geo-Inf. Sci. 2014, 30, 82-87.

29. Jiang, B.; Huang, B.; Lu, F. Spatial Analysis and Geoscience Visualization in GIS Environment; Higher Education Press: Beijing, China, 2002.

30. Duan, J. Spatial Syntax and Urban Planning; South-east University Press: Nanjing, China, 2007.

31. Chen, X.L.; Zhang, L.P.; Zhang, J.; Li, S. China City Statistical Yearbook 2017; China Statistics Press: Beijing, China, 2017.

32. Annual Report on Road Network Density in Major Chinese Cities. Available online: https://www.useit.com. cn/thread-18907-1-1.html (accessed on 4 May 2018).

33. China Vehicle Environmental Management Annual Report 2018. Available online: http://www.gov.cn/ guoqing/2019-04/09/content_5380744.htm (accessed on 9 April 2019).

34. Annual Report on Traffic Analysis of China's major cities in 2017. Available online: https://wenku.baidu. com/view/b167bab0988fcc22bcd126fff705cc1755275fce.html (accessed on 25 January 2018).

35. He, H.; Yang, J. Research on intensive use of urban functional area construction land based on spatial correlation and hotspot analysis. Land Resour. Inf. 2018, 8, 42-49.

36. Liu, J.M.; Hou, X.H.; Wang, Z.Q. Study on the spatial correlation characteristics of cultivated land fragmentation and intensive use of cultivated land. China Land Sci. 2017, 31, 51-59.

37. Gao, X.C.; Li, T.; Cao, X.S. Spatial fairness and changes in transport infrastructure in the Qinghai-Tibet plateau area from 1976 to 2016. Sustainability 2019, 11, 589. [CrossRef]

38. Nolè, G.; Lasaponara, R.; Lanorte, A.; Murgante, B. Quantifying urban sprawl with spatial autocorrelation techniques using multi-temporal satellite data. Int. J. Agric. Environ. Inf. Syst. 2014, 5, 19-37. [CrossRef]

39. Anselin, L. Local indicators of spatial association-LISA. Geogr. Anal. 1995, 27, 93-115. [CrossRef]

40. Ashley, N.A. Demography, foreclosure, and crime: Assessing spatial heterogeneity in contemporary assessing spatial heterogeneity in contemporary. Demogr. Res. 2012, 26, 449-488.

(C) 2019 by the authors. Licensee MDPI, Basel, Switzerland. This article is an open access article distributed under the terms and conditions of the Creative Commons Attribution (CC BY) license (http://creativecommons.org/licenses/by/4.0/). 